\title{
HOW TO RUN AN EFFICIENT EYE CARE PRACTICE DURING AND AFTER THE COVID-19 PANDEMIC
}

\author{
Christina N Grupcheva, Mladena N Radeva, Dimitar I Grupchev \\ Department of Ophthalmology and Visual Sciences, Faculty of Medicine, \\ Medical University of Varna
}

\begin{abstract}
INTRODUCTION: The COVID-19 pandemic is the defining global health crisis of our time and one of the greatest challenges the world has faced. During the pandemic there have been significant changes in the healthcare systems, especially in the access to local eye care. That is why it is essential for any eye care practitioner to adopt the proper measures in order to run a successful and efficient eye care practice during and after the COVID-19 pandemic.

AIM: The purpose of this article is to share expertise on successful re-opening of eye care practice after the peak of the COVID-19 pandemic, providing best protection for the patients, practitioners and support staff.

MATERIALS AND METHODS: Analysis of the possible safety measures and their implementation during the challenging period of re-establishing standard eye care practice after pandemic peak has been conducted.

RESULTS: A clear guidelines regarding organization, policy and leadership are provided after their pilot testing. Special solutions include: environment, attire, behavior, media (web-based behavior), communication with patients, how to manage new patients, what to change for better experience and how to improve leadership during the pandemic situation.

CONCLUSION: Taking proper care for each patient and team member during the difficult times of a pandemic is an investment in the future. An eye care practitioner and practice manager must always be informed, flexible and adaptable, especially in challenging situations such as a pandemic.
\end{abstract}

Keywords: COVID-19, eye care practice, guideline, leadership, ophthalmology

\section{INTRODUCTION}

The COVID-19 pandemic is the defining global health crisis of our time and the greatest challenge the world has faced since World War II. People all around the world follow new rules and have a differ-

Address for correspondence:

Mladena Radeva

Faculty of Medicine

Medical University of Varna

55 Marin Drinov St

9002 Varna

e-mail: mladenaradeva@gmail.com

Received: May 25, 2020

Accepted: June 10, 2020 ent lifestyle. During the pandemic there have been significant changes in the healthcare systems, especially in the access to local eye care. That is why it is essential for any eye care practitioner to adopt the proper measures in order to run a successful and efficient eye care practice during and after the COVID-19 pandemic.

\section{WHAT IS COVID-19}

Coronavirus disease 2019 (COVID-19) is an illness caused by a novel coronavirus now called severe acute respiratory syndrome coronavirus 2 (SARSCoV-2) (1). The virus was first identified among an outbreak of respiratory illness cases in Wuhan City, Hubei Province, China (2). The World Health Organization declared the COVID-19 outbreak a glob- 
al health emergency on January 30, 2020. Since then, the epidemic has escalated and rapidly spread around the world and on March 11, 2020 COVID-19 was determined as global pandemic (3). By March 2020, the European region had become the epicenter of the pandemic with over $40 \%$ of globally confirmed cases (4).

By this moment, WHO reports more than six and a half million confirmed cases with approximately four hundred thousand confirmed deaths and two hundred and sixteen countries, areas or territories with cases (4).

The first COVID-19 cases were associated with a seafood market in Wuhan, initially suggesting a direct food source transmission pathway, but since that time, the person-to-person transmission has been established as one of the main mechanisms of COVID-19 spread. The models of transmission have been identified as host-to-human and human-to-human. There is evidence that environmentally mediated transmission may be possible, so that COVID-19 patients could be acquiring the virus through contact with abiotic built environment surfaces (5). The incubation period for COVID-19 is on average 5-6 days, however, it can be up to 14 days $(3,6)$.

The clinical presentation is generally that of a respiratory infection with a diverse range of severity from a mild cold-like illness to a severe viral pneumonia leading to acute respiratory distress syndrome that is potentially fatal. Characteristic symptoms include cough, fever, dyspnea, muscle aches, sore throat, unexplained loss of taste or smell and even diarrhea. Asymptomatic cases have been reported. Complications of the severe form of the disease include, but are not limited to, multi-organ failure, septic shock, and blood clots (7).

There are limited reports suggesting that COVID-19 can cause conjunctivitis and possibly be transmitted by aerosol contact with the conjunctiva (8). Tear and other conjunctival secretions can potentially be infected by the virus, however, less than $3 \%$ of COVID 19 positive patients have infection of the conjunctiva. Many of the diagnostic and therapeutic methods in ophthalmology require close contact with patients, thereby increasing the risk of exposure (9). Eye care practitioners might be more exposed to the infectious hazard. Several case reports have doc- umented the spread of COVID-19 to ophthalmologists during routine diagnosis and treatment (10).

In a recent interview Alice C. Lorch stated that much of what practitioners do in ophthalmology is elective, so the numbers of patients have "dropped sharply" (11). Ophthalmology may not seem to be on the front line of the coronavirus response, but in fact eye care practitioners sometimes are the first who are in contact with COVID-19 patients. There are a lot of health and economic consequences and the ophthalmology society needs to find the answers of many questions.

Regardless of that so far we are convinced that COVID-19 does not cause specific eye damage, the damage by the virus has had a significant impact on all aspects of eye care.

\section{AIM}

The purpose of this article is to share expertise on successful re-opening of eye care practice after the peak of the COVID-19 pandemic, providing best protection for the patients, practitioners and support staff.

\section{MATERIALS AND METHODS}

During this pandemic, the ophthalmology practice was limited to only emergency cases. That left a lot of time for reorganization and rethinking, as well as an analysis of the possible safety measures and their implementation during the challenging period of re-establishing standard eye care practice after pandemic peak. To provide guidelines, the most important points in the management of the eye care practice were classified into several groups. This arrangement facilitated provision of a step-by-step algorithm for safety and efficiency in the transition period. Those steps we tested in smaller outpatient and bigger hospital-based practice.

\section{RESULTS}

The results are based on pillar questions as follows:

1. How to utilize the time when the practice is not busy

As a manager of an eye care practice, the first action to take in conditions of a COVID-19 infection is the re-organization (staff shifts and protection, economic issues). In our dynamic world, the use of 
digital technologies makes it possible to have phone and web-based presentations and switch to online learning and education. Another clever move is so perform all cleaning and repair work for which in normal conditions there is usually time restriction. Brainstorming, new ideas, and positive changes are essential for creating new materials and organizing new services. Last but not least, staff training is crucial for running better eye care practice.

\section{What happened and what to expect?}

A pandemic happens suddenly and there is no tome for planning. In the case of COVID 19 we experienced a lot of challenges. The most important points are:

$\diamond$ Medical emergency. Limiting close physical contact is key to helping reduce the spread of the coronavirus. The American Academy of Ophthalmology has recommended that ophthalmologists restrict seeing patients to urgent or emergency cases, as well as screening any patients coming into their offices for symptoms of the novel coronavirus. This does not mean that some cases must be postponed. It is extremely important to inform the patients that they must visit an ophthalmologist as soon as possible in situations like eye injury, new floaters or flashes, sudden loss of vision, need for regular eye injections in cases with AMD and diabetic retinopathy, changes in vision, or red eye or eye pain, especially if associated with headache, nausea or vomiting.

$\diamond$ Lockdown, social isolation and high street practices \& stores closed. While physical distancing may be the most effective way of preventing the spread of the virus, this measure may be associated with a range of adverse psychological effects, including fear, anxiety, and depression. This could affect patients and staff. Psychosis is one mental health condition that requires specific attention as it could impact the quality of life and the productivity (12).

$\diamond$ Internet "developed as panacea". Cyberchondria (compucondria) is the unfounded escalation of concerns about common symptomology based on a review of search results and literature online (13). People search information, which pushes them to anxiety and distress. One study found that the cyberchondria during the lockdown is one of the factors are related coronavirus anxiety (14).

$\diamond$ Planned eye care visits decreased four to ten times. The reduction of the work volume could have some negative impact on the psychological state of the practitioners.

$\diamond$ Online consultations. It may be useful to replace routine check-ups with virtual remote consultations long after the pandemic.

$\diamond$ Hospital practices also provided limited eye care. The consequences are delay on the diagnosis and treatment of a new disease, and exacerbated chronic problems. Many patients were reluctant to visit a doctor during the epidemic situation, neglecting the eye health problems. As a result, complications increased.

$\diamond$ Scared and suspicious patients and staff. The psychological warfare waged by COVID-19 has been overwhelming, both for patients and for health care providers. Patients are afraid of contracting and transmitting the virus. Health care staff seems to be susceptible to the stress of uncertainty and loss of control engendered by the COVID-19 situation.

\section{Contamination}

The biggest fear for all parties is contamination. It is essential to step up infection control measures during the COVID-19 pandemic. Thousands of healthcare workers in China were infected with COVID-19 and six of them passed away (15). One of them was Dr. Li Wenliang, a Wuhan ophthalmologist. In early January, he was infected with COVID-19 from asymptomatic glaucoma patient and died from the disease one month later. The close contact between an ophthalmologist and a patient during the eye exam, and the characteristics of different ophthalmic diagnostic and therapeutic procedures, are a potential risk factor for COVID-19 transmission (16).

There are different strategies of preventing COVID-19 transmission in the eye care practice. The possible transmission can have 4 pathways: patient to patient, patient to staff, staff to patient, and staff to staff. This should be considered in every practice, regardless of the size or intensity of work. Therefore, 
several special steps must be followed as per considerations listed below.

4. How to train the staff for the special situation: environment, attire, behavior, media (webbased behavior)

The staff of the practice, without exclusions, must be familiarized with the general guidelines regarding attire, behavior, and communication. Uniformity is a key word in such situation. It is very important that behavioral model is followed by each and every member regardless of the position and seniority. Several guidelines regarding the special situation are provided in Tables 1, 2, and 3 .

\subsection{Media and web-based behavior}

It appears that in sudden lockdown, phone support, out-of-hours voice mail offer and referral to the e-mail options are the first steps to manage a modern ophthalmic practice. Some practices are more and other less prepared for "virtual"/telemedicine consultations. The options over the phone are limited, but they can be improved by video chat on a computer or smartphone. Eye care is not the most suitable specialty for virtual diagnosis, but one important point is that all consultations must be reinforced by written professional advice. Some small gestures, such as free delivery of goods, may show to the patient how valuable they are to a certain practice.

\section{Practice rules}

Practice rules could be classified into 5 groups: patient-related, staff-related, procedure-related and compliance with the general rules. Those are formulated as below:

Table 1. Requirements to staff attire in a pandemic situation: good and bad behaviors

\section{GOOD BEHAVIOR}

White coat

Covered hair

Disposable mask

Dioptric looking glasses/shield

Less jewelry, no rings and bracelets

Short nails, no nail polish

Special indoor shoes

\section{BAD BEHAVIOR}

Long hair down or ponytail

Handmade reusable mask

Protective goggles

Usual jewelry with lots of elements

Artificial nails with decoration

High heels

Table 2. Requirements to staff environment in a pandemic situation: good and bad practices

\section{GOOD PRACTICE}

Protective “sneeze guard”, enough space

Disinfection after each patient

One patient at a time

One staff takes care for all steps

Age consideration for patient slots

Limit the accompanying person(s)

Strictly apply screening procedure

\section{BAD PRACTICE}

Open reception with many staff

Pen and other reusables for all patients

Queues with many patients

Multiple staff for each step

Assign all patients randomly

All support family is welcome

As patient wears mask all should be fine 
Table 3. Treatment of the environment and equipment in a pandemic situation: good and bad practices

Hand washing before and after

Disinfection of all equipment and handles

Use disposables when possible

Separate the rubbish according to the infection risk

Highlight verbally the procedure
Gloves and disinfectant is enough

Chin rest paper stipes are enough

Save the environment and disinfect

Separate the rubbish according to the recycling rules

Patient see it by themselves

\subsection{Patient-related rules:}

Patient-related rules are the first step to ensure patient safety and security. They must be properly communicated and be equal for each patient. The main pillar are as follows:

$\diamond$ Patients/parents must be informed in advance via telephone call, text message, e-mail;

$\diamond$ The rules must be published on the web page and displayed in the practice;

$\diamond$ The rules must be clear and clearly demonstrated by the staff. It is important not to forget that rules are for everyone;

\& The rules must be reasonable and flexible, depending on the situation.

\subsection{Staff-related rules:}

Staff is the key player in each practice, example for precision, but one should not forget that the staff is prone to be infected and transmit the infection. The staff also has suffered a psychological impact by the pandemic and most of the staff members are in complicated situation where they should take and manage risks. The rules to the staff must be:

$\diamond$ Clear and well demonstrated. Educational seminars must be performed regularly. If the manager requires strict compliance with the rules, they must be demonstrated properly.

$\diamond$ Provision of all materials required. Various ophthalmology and health associations are constantly updating their recommendations, so they should be regularly monitored and implemented into clinical practice.

$\diamond$ Team updating and regular training, proactive encouragement and monitoring of the compliance with the rules are essential actions that provide satisfactory results.

\subsection{Procedure-related rules:}

Eye examinations are very specific, and the examiner is often within the intimate space of the examined patient. This not only violates the rules for social distancing but also is associated with risks of compromise during the examination. There are simple measures that are usually taken in a standard situation, but those should be reinforced:

$\diamond$ Frame/phoropter disinfection in between each patient (with gloves).

$\diamond$ During the slit-lamp examination use a slit lamp protector with size as large as possible while not interfering with the quality of the examination.

$\diamond$ Selection of proper technology. For example, use indirect ophthalmoscopy instead of direct ophthalmoscopy.

$\diamond$ Disposable lenses/lens disinfection in between each patient (with gloves).

$\diamond$ New patients training is crucial. The patient must be instructed on how to behave during the exam and what to expect in order to reduce the duration of the eye exam and the possible contact.

The special protective shield is crucial for examination during the pandemic. Our team developed an easy-to-clean protector with wider coverage that was distributed as charity to all practices in Bulgaria (Fig. 1). The most important rule regarding the protector is disinfection after each patient, therefore we work with a minimum of 2 protectors per slit lamp in order to provide proper disinfection. 


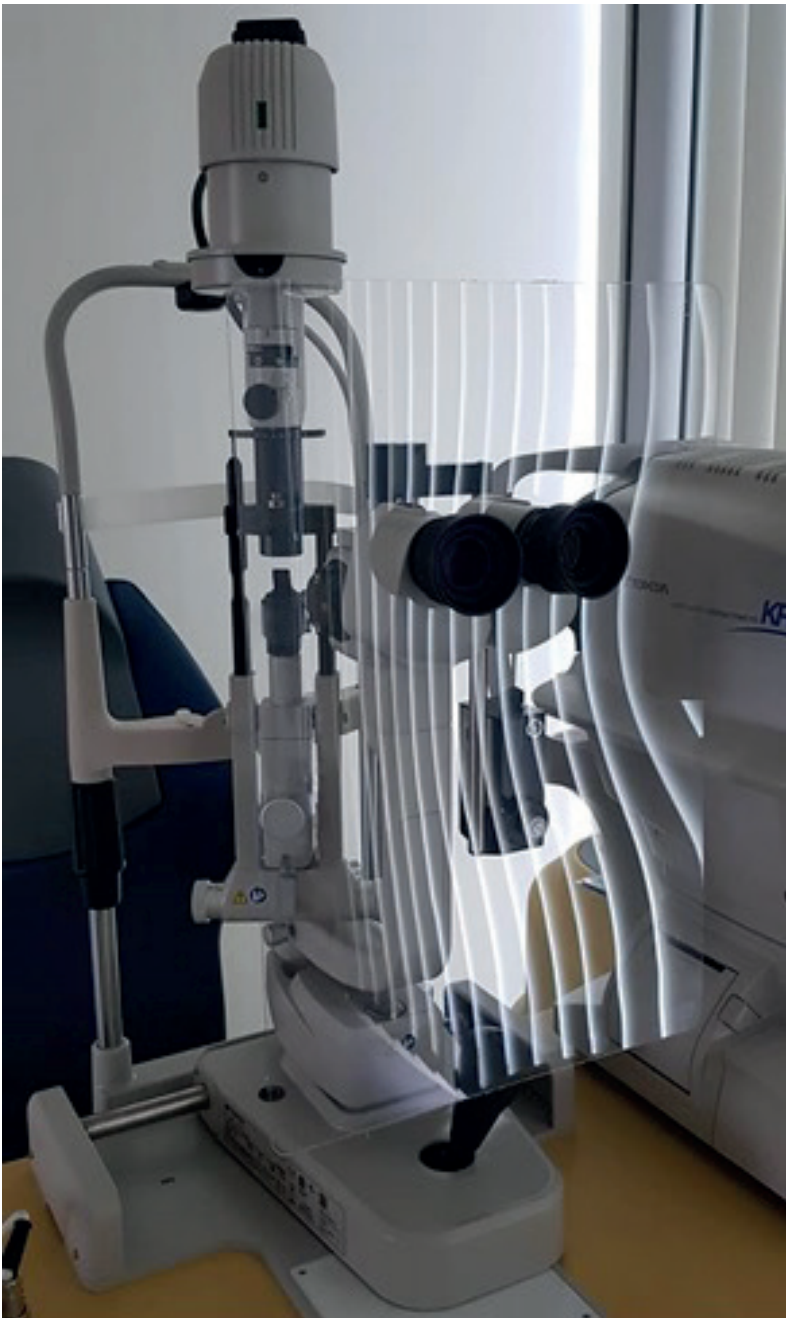

Fig. 1. Protective shield for biomicroscope, providing full separation between the patient and the doctor.

\section{To adapt or not to adapt contact lenses?}

Regarding eye care practice, one of the main problem is unreasonable fear that contact lenses might be a vehicle for viral transmission. There is no scientifically proven information on virus persistence on hydrophilic materials (soft contact lenses) or on other polymers (used for rigid gas-permeable contact lenses). The lack of evidence from previous outbreaks of coronavirus disease, including SARS, suggests that the risk of developing COVID-19 from contact lens wear is low (17). However, eye care practitioners must assess the risk of potential contamination and the intrusion of the quality of life of the patients if they cease to wear their contact lenses.
Considering contact lens wearers, it is important to highlight that those patients are best trained to maintain proper hygiene. Of course, additional measures must be considered and reminded to all contact lens wearers, but it does not look reasonable to stop wearing contact lenses, as glasses are not better perspective for protection. Moreover, change to glasses in a pandemic situation may mean older or insufficient correction, poorer vision and negative impact on quality of vision and life.

New fits after the pandemic should be considered, especially for patients that are seeking new experiences. The new contact lens wearer must follow all rules in order to protect themselves from contamination. Proper hand washing is required before and after fitting, and after removing the contact lenses. Hand washing should be done according to the rules. Daily disposable contact lenses are advisable if possible.

\section{To operate or not to ...}

During the pandemic, planned visits were terminated and all practices operated only in case of emergency. Non-urgent elective services and operations were postponed in order to reduce the risk of transmission. All "high-speed" procedures were thought to be more risky and procedures such as vitrectomy required special measures. Procedures requiring general anesthesia (GA) were accepted as very risky and performed only if no other option existed. Endotracheal intubation was proven as a risk factor for spreading of COVID-19. In the published literature there was suggestion that an ophthalmologist should ensure that a COVID-19 test is done in case GA is required. In all suspicious cases it was postulated that all staff must wear protective eyewear, gowns, and N95 masks (16). One elegant option for protection is utilization of the newest technology such as 3D microscopy. The principle advantage is the head position of the surgeon away from the operative field and also the possibility for better protection.

After the pandemic, ophthalmology patients are still reluctant to be operated. Some patients prefer to wait, but this is associated with complications. In our practice, where we usually perform 300 planned surgeries with 5-6\% emergency cases per month, in May we encountered an increase to 64 patients with 


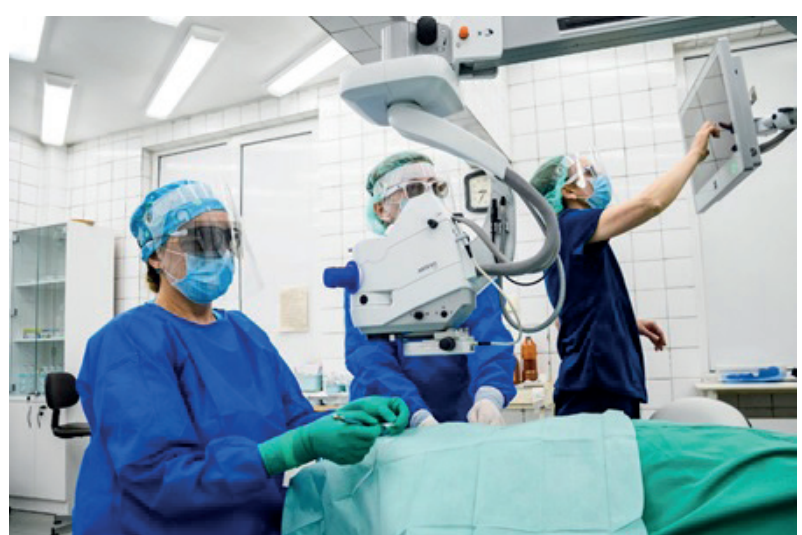

Fig. 2. Utilization of the newest $3 D$ technology in ophthalmic surgery - an option for better protection

glaucoma, cataracts, retinal vascular accidents, and even untreated retinal detachments. This changes the structure of the practice and decreases chances for successful surgery.

8. How to contact and approach patients, how to deal with them

The pandemic disrupted the usual communication at many levels; that is valid for the communication between the patient and the eye care practitioner. Sometimes patients could be so scared and "forget about the eye problem", some of them may have "more serious issues" and postpone the eye care and others could be "advised" that eye problems might wait. Many of them could be worried about the future financial prospective. Many patients could present with eye emergency or complication, because they were afraid to visit an eye care physician. The most important step in such cases is not to try to imply guilt. The support staff must be proactive, showing confidence that all the best will be done. It is essential to give the patient detailed information, ask about any concerns and provide Information about any possible fast and safe solutions, and it is recommended for a service "package" to be provided if possible.

The eye care team must understand the isolation and what they can do for their potential new patients. It must be noted that most people have been isolated, but social contact is important for them. Some of them are prone to worry about new experiences, about the future or have financial concerns.
That is why it is extremely important to perform individualized approach and treatment to every patient.

\section{What to change for better clinical practice?}

The most important steps in improving any clinical practice is having sustainable digital environment, staff coherence, communication within the practice, communication with patients, performing a SWOT analysis, and understanding the dynamic economic changes. One important issue is that money is short and therefore investments are not feasible. However, a good manager should understand that investment in the staff is the best that can be done in such situation. The best leader must keep his precious staff and develop identification with the practice and must know that a pandemic situation is the right time to reduce people of less use. Every study and research possibility must be encouraged and special time must be spent on training junior members. Keeping the positive atmosphere and optimism is one of the main goals. And last but not least, the best strategy is to invest in a good staff member.

\section{KEY POINTS}

$\diamond$ The best option during a lockdown is to utilize the time - every minute is investment;

$\diamond$ After the pandemic the world might be different - visual needs are and will be the same;

$\diamond$ The staff is the mirror of the practice reciprocity is very important for all rules and procedures;

$\diamond$ The patient is in a delicate situation, be a good psychologist try to help not criticize;

$\diamond$ Try to offer "new experiences" - do a "face lift" of your practice;

$\diamond$ Invest wisely - good staff cannot replace the newest equipment but can keep the patients;

$\diamond$ Leadership is difficult but a good leader should enhance the positive effect and cope with the negative consequences.

\section{CONCLUSION}

Taking proper care of each patient and team member during the difficult times of a pandemic is investment in the future. An eye care practitioner and practice manager must always be informed, flexible and adaptable, especially in challenging situations such as a pandemic. 


\section{REFERENCES}

1. Cennimo DJ, Bergman SJ. Coronavirus Disease 2019 (COVID-19) Clinical Presentation. Medscape. 2020. Available from: https://emedicine.medscape. com/article/2500114-clinical.

2. Zhu N, Zhang D, Wang W, Li X, Yang B, Song J, et al. A novel coronavirus from patients with pneumonia in China, 2019. N Engl J Med. 2020; 382(8):727-33. doi:10.1056/NEJMoa2001017.

3. Sohrabi C, Alsafi Z, O’Neill N, Khan M, Kerwan A, Al-Jabir A, et al. World Health Organization declares global emergency: A review of the 2019 novel coronavirus (COVID-19). Int J Surg. 2020;76:716. doi: 10.1016/j.ijsu.2020.02.034.. doi:10.1016/j. ijsu.2020.02.034.

4. WHO. Novel Coronavirus (2019-nCoV) Situation Report - 1. WHO Bull. (2020). Available from: https:/www.who.int/docs/default-source/ coronaviruse/situation-reports/20200121-sitrep1-2019-ncov.pdf

5. Dietz L, Horve PF, Coil DA, Fretz M, Eisen JA, Van Den Wymelenberg K. 2019 Novel coronavirus (COVID-19) pandemic: Built environment considerations to reduce transmission. mSystems. 2020 Apr 7;5(2):e00245-20. doi: 10.1128/ mSystems.00245-20.

6. WHO. COVID-19 situation report 29. Available from: https://www.who.int/docs/default-source/ coronaviruse/situation-reports/20200218-sitrep29-covid-19.pdf?sfvrsn=6262de9e_2.

7. Yi Y, Lagniton PNP, Ye S, Li E, Xu RH. COVID-19: what has been learned and to be learned about the novel coronavirus disease. Int J Biol Sci. 2020;16(10):1753-1766. doi: 10.7150/ijbs.45134.

8. Ankita, Kaur A, Saxena S.K. COVID-19: An ophthalmological update. In: Saxena SK, editor. Coronavirus Disease 2019 (COVID-19). Springer, Singapore; 2020. pp. 81-93. doi:10.1007/978-981-15-4814-7_8.

9. Hong N, Yu W, Xia J, Shen Y, Yap M, Han W. Evaluation of ocular symptoms and tropism of SARSCoV-2 in patients confirmed with COVID-19. Acta Ophthalmol. 2020. doi: 10.1111/aos.14445.
10. Li X, Wang M, Dai J, Wang W, Yang Y, Jin W. Novel coronavirus disease with conjunctivitis and conjunctivitis as first symptom: Two cases report. Chinese J Exp Ophthalmol. 2020; 38(4). doi: 10.0376/ cma.j.cn.115989-20200303-00133.

11. Parry NM. American Academy of Ophthalmology emite actualizaciones sobre COVID-19 para oftalmólogos. Medscape. 2020. Available from: https://espanol.medscape.com/verarticulo/5905159.

12. Brown E, Gray R, Lo Monaco S, O’Donoghue B, Nelson B, Thompson A, Francey S, McGorry P. The potential impact of COVID-19 on psychosis: A rapid review of contemporary epidemic and pandemic research. Schizophr Res. 2020; S09209964(20)30257-7. doi: 10.1016/j.schres.2020.05.005.

13. White RW, Horvitz E. Cyberchondria: Studies of the escalation of medical concerns in Web search. ACM Trans Inf Syst. 2009; 27(4). doi:10.1145/1629096.1629101.

14. Jungmann SM, Witthöft M. Health anxiety, cyberchondria, and coping in the current COVID-19 pandemic: Which factors are related to coronavirus anxiety? J Anxiety Disord. 2020;73:102239. doi: 10.1016/j.janxdis.2020.102239.

15. WHO. WHO Director-General's remarks at the media briefing on 2019-nCoV on 11 February. Available from: https://www.who.int/dg/speeches/ detail/who-director-general-s-remarks-at-the-media-briefing-on-2019-ncov-on-11-february-2020.

16. Lai THT, Tang EWH, Chau SKY, Fung KSC, Li KKW. Stepping up infection control measures in ophthalmology during the novel coronavirus outbreak: an experience from Hong Kong. Graefes Arch Clin Exp Ophthalmol. 2020;258(5):1049-55. doi: 10.1007/s00417-020-04641-8.

17. Jones L, Walsh K, Willcox M, Morgan P, Nichols J. The COVID-19 pandemic: Important considerations for contact lens practitioners. Cont Lens Anterior Eye. 2020;43(3):196-203. doi: 10.1016/j. clae.2020.03.012. 